\title{
BMJ Open Evaluating the feasibility and acceptability of a co-design approach to developing an integrated model of care for people with multi-morbid COPD in rural Nepal: a qualitative study
}

\author{
Uday Narayan Yadav (D) , ${ }^{1,2}$ Jane Lloyd, ${ }^{1}$ Kedar Prasad Baral, ${ }^{3}$ Narendra Bhatta, ${ }^{4}$ \\ Suresh Mehata, ${ }^{5}$ Mark Harris (i) ${ }^{1}$
}

To cite: Yadav UN, Lloyd J, Baral KP, et al. Evaluating the feasibility and acceptability of a co-design approach to developing an integrated model of care for people with multimorbid COPD in rural Nepal: a qualitative study. BMJ Open 2021;11:e045175. doi:10.1136/ bmjopen-2020-045175

- Prepublication history for this paper is available online. To view these files, please visit the journal online (http://dx.doi. org/10.1136/bmjopen-2020045175).

Received 24 September 2020 Revised 22 December 2020 Accepted 12 January 2021

\section{Check for updates}

(C) Author(s) (or their employer(s)) 2021. Re-use permitted under CC BY-NC. No commercial re-use. See rights and permissions. Published by BMJ.

${ }^{1}$ Centre for Primary Health Care and Equity, University of New

South Wales, Sydney, New South Wales, Australia

${ }^{2}$ Center for Research, Policy and Implementation, Biratnagar, Nepal

${ }^{3}$ School of Medicine, Patan Academy of Health Sciences, Kathmandu, Nepal

${ }^{4}$ BPKIHS, Dharan, Nepal

${ }^{5}$ Ministry of Health and

Population, Kathmandu, Nepal

Correspondence to

Dr Uday Narayan Yadav;

unyadav1@gmail.com

\section{ABSTRACT}

Objective To understand the feasibility and acceptability of a co-design approach to developing an integrated model of healthcare for people with multi-morbid chronic obstructive pulmonary disease (COPD) in rural Nepal.

Settings A rural setting of Nepal.

Participants Data collection included five video recordings, five key informant interviews and observation notes from a final co-design workshop that involved a total of 68 stakeholders: persons with COPD and their family members; healthcare providers, including respiratory physicians; local community leaders; representatives from local, provincial and federal government; academics; and representatives from nongovernment organisations.

Primary and secondary outcome measure(s) Feasibility and acceptability of using a co-design approach to develop an integrated model of care for people with multi-morbid COPD in rural Nepal.

Results Our qualitative evaluation of the Hasso Plattner's co-design process found that all stakeholders (including people with COPD/community members, primary care practitioners and local government/ senior health officials) were actively engaged in and significantly contributed to the process of co-design. Four main themes were identified which determined the feasibility and acceptability of the resulting integrated model of care: engagement of stakeholders, factors contributing to the co-design, consequences of the codesign process, and challenges and opportunities learnt by the researchers and participants in the co-design process. Based on the relationship between the four main themes emerging from this research, we developed an evaluation framework to guide the co-design of a health service innovation.

Conclusion Our study demonstrated the feasibility and acceptability of the Hasso Plattner's co-design process. Our findings suggest that this co-design approach can be useful and acceptableto local communities and government agencies. It enabled the meaningful contribution of adiverse group of stakeholders in the design and delivery of health services in low-income and middle-income countries.

\section{Strengths and limitations of this study}

- This is a unique study that evaluated the feasibility and acceptability of Hasso Plattner's co-design process in developing an integrated model of care for people with multi-morbid chronic obstructive pulmonary disease in rural Nepal.

- It used multiple data sources (interviews, videos and observation notes) to evaluate the co-design process.

- This study resulted in the development of an evaluation framework for a co-design process that can guide researchers/academics, policy-makers and practitioners.

- A robust qualitative analysis using a focused ethnographic method led to the generation of contextually relevant evidence.

- This was a small qualitative study based on purposively selected participants who participated in the co-design workshop and who may have been more likely to report positive views on the process.

\section{INTRODUCTION}

In recent years, co-design, a collaborative change process, has been widely used to develop and improve health services. Co-design methods have been cited as being successful in creating greater awareness of the concerns among the stakeholders, shared ownership of the product, an improvement in care and treatment processes and better health outcomes. ${ }^{1-3}$ A co-design process offers a 'people-centred' solution through the active engagement of patients and their family members, healthcare providers and other stakeholders in all stages of the design. The stages of co-design include exploring the problems, identifying priorities, ideating and finalising solutions tailored to the local context and implementing these solutions with and for, the people for whom it 
is designed, ensuring the results meet their needs and are usable. ${ }^{4}$ A recent summary of literature reviews ${ }^{5}$ highlighted the benefits of co-design in health service research. Several authors underscored the need for the meaningful and active engagement of end users in research to address research waste ${ }^{5-7}$ Co-design plays a crucial role in designing person-centred healthcare solutions by engaging end users in the development and testing process. ${ }^{5}$

Chronic obstructive pulmonary disease (COPD) is an under prioritised health issue in low-income and middle-income countries (LMICs). Limited institutional capacity (such as infrastructure and human resources for health), financial resources and ineffective health leadership in delivering health services contribute to this situation. ${ }^{8}$ Among the LMICs, Nepal is one of the poorer countries in South Asia. It has the highest prevalence of COPD (11.7\%). ${ }^{10}$ In response to this need, we conducted a study among people with COPD between 2018 and 2019 and identified low levels of health literacy, poor self-management practices and various barriers to selfmanagement at patient-family, community and service provider levels. ${ }^{9} 1112$ These findings established the need to engage multiple stakeholders in service design to address the self-management needs of multi-morbid COPD people in Nepal.

The effective engagement of different stakeholders requires careful consideration and effort to ensure that power imbalances are addressed, and participants can make a meaningful contribution to the design of the services. The latter is determined largely by the methodology and tools used in the co-design activities. ${ }^{13}$ Effectiveness of any co-design approach largely depends on the engagement strategy, attitudes, skills, values and relationships between the stakeholders and researchers, engaging content used during the workshops (such as information packs, learning materials and knowledge exchange processes), clarity on the roles of stakeholders, clear communication and a work plan. Published reviews that evaluate the impact of co-design processes have found both positive ${ }^{1214}$ and negative results. ${ }^{15}$ Positive factors include patient empowerment, positive emotions, credibility of knowledge produced, results adapted to local contexts, development of greater empathy and the development of contextually appropriate interventions. Negative factors include the time taken to participate in co-design process and the financial resources required to conduct co-design workshops.

We used a theory-guided co-design process proposed by the Hasso Plattner Institute of design ${ }^{16}{ }^{17}$ at Stanford to design an integrated model of care for delivering selfmanagement interventions for the people with multimorbid COPD in rural Nepal. There are four stages in the process: empathise (understanding the problems), define (defining and prioritising the issues), ideate (designing the solutions) and prototype (refining the solutions). Stakeholders engaged in the co-design process included people with COPD and their family members; healthcare providers, including respiratory physicians; local community leaders; and representatives from local, provincial and federal government, academics and representatives from local and international non-government organisations (NGOs). In the prototype stage, we conducted a 1-hour co-design workshop to finalise our designed prototype. We used multiple engagement strategies, including stakeholder mapping, engagement of active influential social workers and local political leaders at a community level health literacy workshop. Active engagement was achieved by the use of contextual leaning workshops, interpreters and visual diagrams.

Following the workshop, an evaluation of the engagement strategy used in co-design was undertaken to determine what worked well and what did not and how, and in what way, stakeholders subsequently contributed to the outcomes of the co-design process. The use of co-design is commensurate with the principles of working respectfully in LMICs, but the practice is largely untested. Therefore, our study sought to answer the following questions:

1. How well did stakeholders engage in the co-design process?

2. What was the feasibility and acceptability of the codesign process in developing an integrated model of care?

\section{METHODS}

\section{Research design}

This was a qualitative study that used a focused ethnographic approach. ${ }^{18}$ Data collection included video recordings, key informant interviews (KIIs) and overt observation notes from the final co-design workshop. We used interactive evaluation to analyse the data as has been discussed in the implementation science literature. ${ }^{19}{ }^{20}$ It encapsulated different elements that are essential to successful evaluation such as attention to values, beliefs, knowledge, skills, actions, interpersonal and power dynamics in the decision-making process. ${ }^{21}$ This approach is useful when the researcher has background knowledge of the study population, and uses a range of different methods of data collection ${ }^{22} 23$ to generate a holistic description of the phenomenon. Also, integration of different data sources (methodological triangulation) reduced systematic bias, thereby increasing the credibility of the interpretations and derived conclusions. ${ }^{24}$

\section{Sample and setting}

The sample for this study included stakeholders who were engaged in a co-design project to develop an integrated model of care for people with multi-morbid COPD conditions of Sunsari district, Nepal. A total of 68 stakeholders participated in the co-design workshop including: people with COPD ( $\mathrm{n}=10,4$ female and 6 male) and their family members $(n=7)$; healthcare providers, ${ }^{8}$ including one respiratory physician and two primary care doctors; local community leaders $(\mathrm{n}=12)$; and representatives from local government ( $\mathrm{n}=14$ from agriculture, health, 
Table 1 Details on data collection and sampling

\begin{tabular}{|c|c|}
\hline Types of data & Data collection and sampling \\
\hline Video recordings & $\begin{array}{l}\text { The entire workshop was video recorded by a professional videographer. All the participants in the } \\
\text { workshop were informed about the video recordings of the programme and its anonymous use in research } \\
\text { and were asked to provide consent for its use in a programme participant diary. Videos were included } \\
\text { if they met the following selection criteria: (1) video recordings that sufficiently detail and present the } \\
\text { activities of a co-design workshop to refine a model of care, (2) video recordings that capture the discourse } \\
\text { of the key stakeholders, (3) video recordings that connect the participants of being with/being there (social } \\
\text { actions, interactions, roles and positions), and excluded videos of the refreshments, energisers and photo } \\
\text { sessions. In all, eight clips were obtained; however, five were chosen/selected based on the selection } \\
\text { criteria, research questions and phenomena being studied. Videos that were included met the following } \\
\text { selection criteria: (1) video record that sufficiently detailed and presented the activities of a co-design } \\
\text { workshop to refine a model of care, (2) video record that captures the discourse of the key stakeholders } \\
\text { and (3) video record that connect the participants of being with/being there; and were videos excluded } \\
\text { were of the refreshment, energiser and photo session. }\end{array}$ \\
\hline $\begin{array}{l}\text { Key informant } \\
\text { Interviews }\end{array}$ & $\begin{array}{l}\text { Of all the participants of the co-design workshop, five participants were purposively selected based on } \\
\text { their interest to participate in the in-depth interview. All five participants have been involved in the project } \\
\text { since its inception and took an active part in all the activities of the project. These participants were } \\
\text { interviewed by the lead author with the help of an interview guide at the workshop venue. All the interviews } \\
\text { were audio recorded, and note taking was done by the trained research assistant. All five consented to take } \\
\text { part in the in-depth interview conducted in Nepali, which lasted for } 30-40 \text { min. }\end{array}$ \\
\hline $\begin{array}{l}\text { Observation } \\
\text { notes }\end{array}$ & $\begin{array}{l}\text { During the co-design workshop, the observation notes (eg, physical gestures, notes on what was } \\
\text { being said, interactions between the participants, punctuality and participants attention in the co- } \\
\text { design workshop) were taken by two research assistants and one investigator from the research team. } \\
\text { Principal investigator of this study cross-checked the observation notes with research assistants for any } \\
\text { inconsistencies and lack of clarity. }\end{array}$ \\
\hline
\end{tabular}

education and industry), provincial government $(n=4)$, federal government $(n=1)$, academics $(n=3)$, media representatives $(n=4)$ and representatives from local and international NGOs $(n=5)$. All 68 participants were purposively selected in coordination with the local government authorities, which was solely based on the engagement and contribution of the stakeholders in the research. The co-design workshop was a one-day (6 hours) workshop conducted in Nepali language to capture feedback from all the stakeholders on the designed integrated model of care in the community setting (community seminar hall of one rural municipality). The workshop was jointly moderated by the lead author, an academic from Nepal and a local government health coordinator.

\section{Data collection and sampling}

Video recordings, KIIs and observation notes involved in participant interactions at the co-design workshop were collected, and the details are presented in table 1.

\section{Patient and public involvement}

In this study, people with COPD and all potential stakeholders were involved in all stages of the co-design process and the results from the study were disseminated to them through meetings and co-design workshops. All the stakeholders, including people with COPD and their families, were recruited and engaged during the empathise (understanding the problem) phase of co-design. Local government and the advisory group $(n=12$, including patients, specialists, health coordinators, local government representatives and an academic) provided feedback on the application of evidence generated from the co-design process.

\section{Data analysis}

The three sources of data were translated from Nepali into English by the lead author. Table 2 provides the details of the translation. Narratives of video recordings $(n=2)$ and interviews $(n=2)$ were chosen randomly and were checked by two independent bilingual researchers who were not involved in the research in order to ensure the reliability. The three team members (first, second and last authors) conducted regular discussions and reviews to ensure the quality of information, with adjustments made to the vocabularies that caused discrepancies and misapprehension. All the collected data were analysed using thematic analysis guided by Braun and Clarke. ${ }^{25}$ NVivo software (V.12.00) was used to organise the data and facilitate the coding process.

\section{Ethics}

All the participants completed and signed an informed consent form. No monetary incentives or any gift vouchers were provided to the participants for their involvement in the project or qualitative interviews. However, participants did receive reimbursement for travel to attend final co-design workshop.

\section{RESULTS}

The five KIIs included a representative from the local rural municipality, a patient, a policy officer from the 
Table 2 Details on translation

\begin{tabular}{ll}
\hline Sources of data & Translation details \\
\hline Video recordings & $\begin{array}{l}\text { Spoken words and their meaning (interactive process) were explored. Also, visual activities like } \\
\text { talk, gesture, body language, facial expression, gaze, social/cultural identity and engagement } \\
\text { with participants were noted. All the verbal and non-verbal activities were translated into } \\
\text { narrative verbal descriptions (interpretive video analysis). } \\
\text { Social context theory }\end{array}$ \\
& $\begin{array}{l}\text { at whermed our process. This required the researchers to look closely } \\
\text { associations. }\end{array}$ \\
Key informant interviews & $\begin{array}{l}\text { The recordings of key informant interviews conducted in the local language were translated into } \\
\text { English. }\end{array}$ \\
\hline Written observation notes & Observation notes were taken in English, so no translation was required.
\end{tabular}

Ministry of Social Development, a senior health officer from the federal ministry and an academic. They were all aged between 30 years old and 55 years old. The majority were men $(n=4)$ and ethnically diverse with the five participants representing three different Nepalese ethnicities (Madhesi, Indigenous and higher caste).

Four broad themes emerged from the triangulation of the data from the KIIs, videos and observation notes. The themes are engagement of stakeholders, contributors to the co-design process and consequences of the co-design process. In addition, the challenges and opportunities identified by the researcher and participants in the co-design process are summarised. The themes provided the foundation for the evaluation framework for co-design process which is outlined in figure 1 . The framework illustrates the relationship between the three main themes emerging from this research.

\section{Engagement of stakeholders in co-design}

In this theme, we present the contributing factors to stakeholder's engagement, indicators and signs of participant engagement and the impact of engagement in the co-design process.

\section{Contributing factors to stakeholder's engagement}

Participants described various intrinsic and extrinsic factors associated with the active engagement of the stakeholders. Most of the participants cited the level of respect and trust, sense of equality of power between the stakeholders, the realisation of research gaps and benefits from the research, valuing people's ideas, opportunity to learn new things, motivation from researchers and the use of culturally friendly process as the intrinsic factors that facilitated their active engagement in the research process.

“...Well! I decided to join this research because I knew that respiratory health in an untouched topic and engagement in the project would give [me] an opportunity to learn new things that I can use in my professional career." [A Policy Officer, workshop participant]

Participants also reflected on the value of a culturally friendly process and their level of trust in the research.
"I actively participated because the researcher made us [it] clear about the steps of research, and something that was topmost was a level of trust with the researcher [researcher shares culture and uses local language]." [A patient representative, workshop participant]

Some of the extrinsic factors that helped participants to engage in the co-design workshop were a comfortable environment (provision of water and refreshments, the arrangement of bathrooms and the use of an appropriate venues in a convenient location), reimbursement for travel expenses, engagement of active change agents (influential social workers) and accessible information shared in the workshop/meetings. Reimbursement was an important extrinsic factor that motivated the patients and caregivers to actively engage in the co-design activities, and this was also evident in one of the video clips where patients/caregivers were first in the queue to receive the reimbursement for their participation. All the participants highlighted the importance of choosing a local venue for conducting learning activity/workshops.

"I will say, the researcher did really a great job by choosing a venue which encouraged people to participate in the all the activities and the money spent by the researcher remained in community." [An academic, workshop participant]

\section{Signs and benefits of engagement}

In video clips from the co-design workshop, we observed signs of engagement, including punctuality of attendance, gestures (greetings, nodding and smiling) and postures (stretching necks and tracking with their eyes), enthusiastic support for the activity, patience (relaxed position), giving standing ovations to the presenters, note taking, the interaction between the participants, patients and family members sharing their journeys, use of translators (by those who were unable to understand other than their local language like Nepali or English) and attendance throughout all sessions of the co-design workshop. Of these signs, the most notable were punctuality of the participants attendance at the co-design workshop (all participants arrived $30 \mathrm{~min}$ before the co-design 


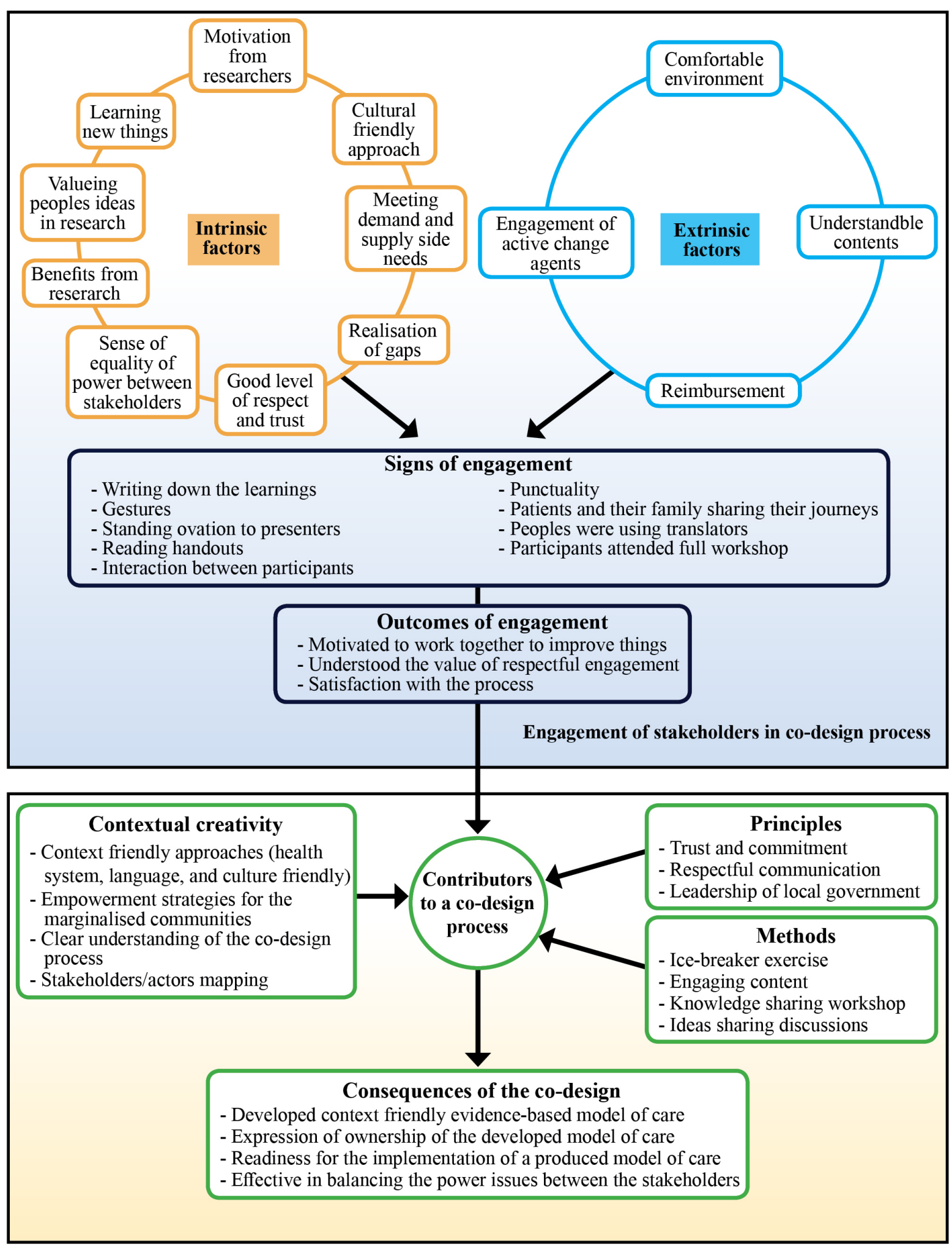

\section{Challenges and opportunities}

- Labour intensive work

- Limited engagement of women from marginalised - Funding challenges community

Figure 1 Framework to guide evaluation of the co-design process. 
workshop) and questions asked by local leaders of the patients and their family members, both of which were rare in the Nepalese context.

Because they were engaged, participants were observed to be motivated as they actively participated in the discussions. Moreover, participants used simple and nontechnical language, were friendly and welcoming and were open and flexible in their views about how to reach collaborative decisions.

"This was the first research where I engaged in all activities of any project. One of the key things that I learnt from this project is to be flexible and receptive to ideas of others... [Laugh]. Also, I came to know how the democratic process works in real research... [Laugh]." [A local government representative, workshop participant]

\section{Contributors to the co-design process}

To understand the factors contributing to the co-design, we analysed both the qualitative interviews and the video clips of the co-design workshop. A summary of these findings is reported below under three sub-themes.

\section{Contextual creativity}

Participants described contextually guided processes of learning, sharing ideas and building the capacity of the stakeholders to make meaningful contributions to refining solutions for their community.

"The researcher gave us a full picture of the opportunity landscape and the steps to design a solution for identified problems at our doorstep by holdings different community meeting. In every activity, they [researchers] showed respect to our culture and identity. Now, most of the people in our village know them [research team]." [A policy officer, workshop participant]

A majority of the participants highlighted the importance of empowerment strategies used in the co-design process. These included community-centred educational sessions on health and development issues, use of local community shops for breakfast and lunch, and knowledge exchange sessions in the local community by involving youth, women, social workers and political leaders.

"Gaining trust and commitment of marginalised communities is not an easy task. The reason why you people [research team] got this [committed participation] is because you all invested your resources in building trust, educating and empowering the people.” [A Senior Health Officer, workshop participant]

Participants also acknowledged the value of using interpreters to address the language barriers, particularly for the patients and their family members who did not sufficiently understand Nepali but spoke other local languages.
"Presence of interpreters (who were fluent in Nepali, local Maithili, Tharu and English language) in the co-design workshop helped us to understand the information." [A patient representative, workshop participant]

A few participants also praised the strategy of networking and engaging influential government officials in the project. This was a very challenging task in Nepal usually requiring a daily cash allowance to enable them to attend (not used in this study).

Some participants noted that the learning sessions had empowered the marginalised and indigenous communities. These were observed in the video clips to be actively engaged in discussions and the idea generating exercises. However, women from marginalised communities were seen to be less engaged than other participants.

Government officials involved in the co-design process appreciated the stakeholders mapping exercise, face-toface meetings and follow-up via phone, sharing meeting minutes and acknowledging the ideas and creativity of government officials in the co-design process.

"Well, now in Nepal, the young implementors like us want to engage in academic research to boost knowledge, which can be useful in bridging the gaps. I believe change is only possible if we [government officials and academics] can create a blended learning environment which can benefit both the academia and program implementors like us." [A policy Officer, workshop participant]

A government representative appreciated the model of care:

"Well, the most remarkable things you [the research team] did is you developed a model of care that is fully aligned with our health care delivery system and policy." [A Senior Health Officer, workshop participant]

\section{Principles of co-design}

Co-design is more than tapping into individual knowledge; it is about identifying and respective shared values and authentically considering diverse viewpoints. Participants indicated that key values included trust, commitment and respectful communication. In this regard, participants appeared trusting and were observed chatting to each other in a video clip of the final co-design workshop. A few participants felt the respectful environment enabled the generation of new ideas.

"The co-design process is very useful in helping put aside professional egos and creating a trustful and respectful environment." [An academician, workshop participant]

Presenters use of an elevator pitch helped participants to understand the importance of their engagement and contribution and this was appreciated by some of the participants. Other participants appreciated being 
consulted about the choice of venue and dates for the co-design activities. Sending the agenda in advance enabled the stakeholders to prepare for the sessions. Ensuring invitations were sent on government letterhead and that phone call reminders were from the communication team of the local government helped participants to feel ownership and commitment to the project.

"Clear communication from the initial stage of design process creates ownership, fosters relationships between all researchers and stakeholders, thus leading to great enthusiasm and involvement in the design of [the] service." [An academician, workshop participant]

\section{Tools and processes of co-design}

Participants appreciated the use of different tools and strategies to increase engagement, learning and interest in the workshop activities. These strategies included the use of name tags for all participants, an opening session acknowledging the culture and tradition of participants, the involvement of a local government official as a facilitator, the ice-breaker exercise, the interactive learning and knowledge exchange workshop, handouts in both English and Nepali, energising sessions, printed reading materials, ideas sharing working groups and a feedback session. When asked what they liked about the workshop, patients noted the ice-breaker exercise, the energiser activity, and interactive learning and discussion sessions.

"I would say all the activities were well planned, which allowed the participants to learn and to contribute actively and creatively." [A local government representative, workshop participant].

Video clips of the workshop showed participants participating in discussions and the ideation activities.

\section{Consequences of the co-design process}

The benefits of co-design were identified by the healthcare providers, academics and policymakers. They described the usefulness of co-design in developing contextually relevant evidence, addressing power imbalances between stakeholders and readiness for implementation. Half of the participants reported the co-design process was likely to support implementation of the service, creating an avenue for translation in other settings. However, these ideas were not discussed by the participant representing the patient group.

"Co-design is a wonderful process that can address the implementation challenges and help to achieve the project goal." [An academic, workshop participant]

In one of the video clips, the local mayor reported being enlightened by the model of care and agreed to provide all the support required for its implementation.

"Well, I am not a health personal nor a researcher...I am a politician. [Laugh] ...but the goal is the same... to serve the community. This research has opened the eyes of those all who want to improve the health care delivery system." [A senior local government representative, video]

\section{Challenges and opportunities}

Implementation of a newly designed solution is hard in any context because of limitations to resources, including money and time. The funding challenges were described mainly by local government and Ministry officials.

"I understand how important this model of care is, but I am not in the position to provide funds because our health budget is low. However, I can commit all other supports [reform in local-level policy, human resources for health, office set up etc.,] needed for Implementation." [A senior local government representative, video]

In the video clips, some women from the disadvantaged communities (particularly members from the Madhesi and Dalits communities) were observed endorsing the opinions of senior health professionals and local government representatives. However, the males belonging to disadvantaged community were observed to be actively participating in the co-design activities.

It is acknowledged that securing the engagement of some stakeholders (particularly government officials) can be difficult to obtain due to the stakeholders competing priorities. Observation notes revealed that researchers held several meetings, follow-up calls and networking processes to ensure the engagement of government officials in the co-design process and it was found to be effective.

\section{DISCUSSION}

Increasingly the co-design process is seen as a central concept in health service improvement. Its purported benefits include the practical application of the principles of social democracy and justice, identifying shared priorities and improving services with and for the benefits of the user group. ${ }^{26}$ The increasing adoption of the co-design process has resulted in a need to evaluate what is feasible and acceptable in this process. The present study evaluated the co-design process used in developing an integrated model of care to deliver self-management support for the people with multi-morbid COPD in rural Nepal.

\section{Engagement and its contributing factors}

In our study, we found the successful engagement of the stakeholders (including users/community members, primary care and local government/senior health officials) and the contextual creative processes to be the contributors to an effective co-design process. Effective and active engagement of the stakeholders is a central point that should be emphasised in any co-design process. Active engagement of stakeholders in a co-design process is largely dependent on both intrinsic and extrinsic factors. 
While many studies have sought to involve a wide range of stakeholders in the design of interventions/services, only a few studies ${ }^{27-30}$ have explored the reasons behind engagement in the co-design process. These studies reported a limited number of intrinsic factors (eg, belief among the stakeholders that they can contribute, feeling honoured, maintaining regular contact with stakeholders understanding needs, relationships and trust) and extrinsic motivators (vouchers, snacks and refreshments) for engagement in co-design process. It is recognised that there are a lack of effective engagement strategies and a need to better understand the factors influencing stakeholder engagement. ${ }^{31}$ To address this gap, we identified various additional intrinsic and extrinsic factors (as explained in figure 1) that influence the stakeholder's engagement in developing countries like Nepal.

\section{Engagement signs in co-design}

The challenge in the co-design process is to empower and actively engage diverse stakeholders. Fruitful engagement is underpinned by the principles of social democracy, where all stakeholders are respected as equal associates contributing their expertise in the design of services. Engagement of stakeholders can be measured by noting physical signs of engagement such as smiles, gestures and interactions (figure 1), which have not been previously reported in published health service co-design studies. In particular, our experience suggests the value of using video data to observe the signs of stakeholder engagement in co-design research. Several authors ${ }^{52} 33$ have suggested a need for documentation of a rich description of engagement outcomes. In this regard, our study identified motivation to work together, understanding of the value of the co-design process and satisfaction with the strategy, tools and techniques as engagement outcomes. Overall most of the challenges identified in the previous studies ${ }^{30} 34$ were addressed in the present study.

\section{Co-design process: moving from consultation to partnership with stakeholders}

Co-design is a non-linear democratic process in the design of the solutions which fit the context and empower the stakeholders while designing solutions. ${ }^{35} 36$ An integrated model of care was developed in line with the policies and practices of the healthcare delivery system of Nepal. Previous studies have highlighted the need for innovations to fit existing organisational systems (policy and healthcare delivery system) in order to achieve sustainability. ${ }^{37}$ The implementation of the designed solutions and also dissemination by local government of such evidence-based practices in their network or at policy forums can thus be seen to as outcomes of the co-design of health policies and new organisational structures. Our study demonstrated that empowerment and educational activities adapted to the local language and culture could facilitate trust, confidence, empowerment and a sense of inclusiveness among the stakeholders. Several ${ }^{39-41}$ studies have recognised approaches fitting to culture and language' as important factors in active involvement of stakeholders in the co-design process. Achieving the trust and commitment of the stakeholders is a key requirement for the transformation of health systems in LMICs. ${ }^{42}$ This can be earned by respectful communication from the beginning of the co-design process as has been documented in the previous studies. ${ }^{41444}$ Our findings highlight the importance of tailoring the co-design processes to the local context, particularly in LMIC like Nepal, with a diversity of local language/s and culture.

Tools and processes for generating engagement in co-design and adaptation to local context

The interest and ideas of the stakeholders can be elicited using the tools and processes for generating engagement in co-design processes such as community learning exercises, knowledge sharing workshops, ideas sharing workshops and decision-making methods. The most typical operational methods ${ }^{5} 45$ in co-design according to the published evidence included focus group discussions, interviews and surveys. Less frequently mentioned were town meetings, symposiums and workshops. ${ }^{46}$ In this regard, evidence ${ }^{47}$ suggests the clear need to report context-specific, tailored, operational methods in co-design research. In the Nepalese context, these tools and processes had been rarely used previously when designing a health service intervention. Our findings provide a guide for co-designing health service intervention through using contextual engagement tools in countries like Nepal.

\section{Acceptability and feasibility of model of care}

Our results demonstrated that the co-design process proposed by the Hasso Plattner Institute of design was acceptable and feasible in the local context. While leadership plays an important role in championing a global health intervention, commitment from local leaders to achieve health goals is crucial. In our study, the commitment of local leaders and other stakeholders helped to create an enabling environment for the design and implementation of an integrated model of care. For example, stakeholders reached consensus, on modifications to the model of care, and expressed ownership and readiness to implement it at the local level. In support of our findings, previous co-design literature has described a range of benefits, including grounding the intervention in the real-world experience and developing people-friendly communication tools for clinical practice. ${ }^{48-50}$ Research from health services and implementation science has consistently highlighted the failure of translation of interventions into the practice setting. ${ }^{51}{ }^{52}$ This is called the 'improvement-evaporation effect' by National Health Service in the UK ${ }^{50}$ Implementation is often prevented by lack of active involvement of marginalised communities in the design process and lack of funding for implementation. ${ }^{342}$ These lessons were reflected in our findings.

Our results suggest that the prototyping stage of the co-design process was feasible and acceptable to 
stakeholders. Further refinements may be made during the implementation phase of model of care. From our experience of this co-design process, we would recommend the use of co-design to develop people-centred solutions aimed at improving healthcare delivery in LMIC such as Nepal.

\section{Strengths and limitations of research methods}

Our methodology involved the use of diverse methods of data collection, including videos, interviews and observation notes, to provide a holistic description of the studied phenomenon. This methodology has not been previously used in an evaluation of the co-design process for health service development. We believe this increases the rigour and credibility of the evaluation. Moreover, a robust qualitative analysis using a focused ethnographic approach by one investigator (with input from Australian academics), sharing the culture and language of the study setting, led to the generation of findings with a contextual lens.

A limitation of the study method was the purposive selection of participants. This may have influenced their reaction to the co-design process. Additionally, interviews were conducted by one member of the investigator team who had good rapport with all the stakeholders, and this may have influenced participants' views.

\section{CONCLUSION}

The results of this study support the growing evidence that co-design is a useful process in health service development. We found that it helped to create ownership among the stakeholders and paved the way for implementation in the real-world settings. The co-design process from Hasso Plattner Institute of design appeared to be both feasible and acceptable in designing an integrated model of care to deliver self-management interventions for people with multi-morbid COPD in rural Nepal. This study also resulted in the development of an evaluation framework for a co-design process that can guide researchers/academics, policy-makers and practitioners. Evaluation of its application in other LMICs is required.

\section{Twitter Uday Narayan Yadav @UNYadav}

Acknowledgements We would like to thank all the people with chronic obstructive pulmonary disease, family member's community representatives, local government representatives, representatives from local community-based organisations, district level non-government organisation (NGO) and international NGO representatives, political leaders, healthcare professionals, advisory committee members and academics, representatives from policy and implementation units of state and federal level government. A special thanks goes to Mr Shree Prasad Yadav, Mr Sagar Parsai, Mr Bhogendra Dotel, Dr Roshan Pokharel, Mr Sagar Dahal, Mr Anil Dev, Dr Laxmi Narayan Yadav, Mr Krishna Deo Yadav, Mr Gajendra Yadav and many other health professionals from the Federal and Province Ministry, including media representative who supported in this endeavour. Also, I would like to acknowledge Sarah Ford (an administrative staff member at Centre for Primary Healthcare and Equity, University of New South Wales), Dr Cathy 0' Callaghan (research fellow) and all other Centre for Primary Health Care and Equity staffs for their all support in this research. Moreover, I would like to acknowledge Dr. Hassan Hosseinzadeh (School of Health and Society, University of Wollongong) and PhD review panel members: Professor Evelyne de Leeuw and Associate Professor Bette Liu for their constructive feedbacks in this project.
Contributors UNY conceived and conducted the fieldwork and wrote the first version of this manuscript as a PhD student under the supervision of $\mathrm{MH}, \mathrm{JL}, \mathrm{KPB}$, NB and SM. UNY participated in the data analysis and interpretation of findings under the supervision of $\mathrm{JL}$ and $\mathrm{MH}$. All authors read and gave their approval to the final version of this manuscript submitted for publication.

Funding The authors have not declared a specific grant for this research from any funding agency in the public, commercial or not-for-profit sectors.

Competing interests None declared.

Patient consent for publication Not required.

Ethics approval This research received ethics clearance from Human Research Ethics Committee of University of New South Wales, Sydney, Australia (HC180502), and the Nepal Health Research Council (Reg no 544/2018). All the participants completed and signed an informed consent form.

Provenance and peer review Not commissioned; externally peer reviewed.

Data availability statement No data are available. Data sharing not applicable as no datasets generated and/or analysed for this study. The de-identified data are available on request from the corresponding author.

Open access This is an open access article distributed in accordance with the Creative Commons Attribution Non Commercial (CC BY-NC 4.0) license, which permits others to distribute, remix, adapt, build upon this work non-commercially, and license their derivative works on different terms, provided the original work is properly cited, appropriate credit is given, any changes made indicated, and the use is non-commercial. See: http://creativecommons.org/licenses/by-nc/4.0/.

\section{ORCID iDs}

Uday Narayan Yadav http://orcid.org/0000-0002-6626-1604

Mark Harris http://orcid.org/0000-0002-0705-8913

\section{REFERENCES}

1 Manafo E, Petermann L, Mason-Lai P, et al. Patient engagement in Canada: a scoping review of the 'how' and 'what' of patient engagement in health research. Health Res Policy Syst 2018;16:5.

2 Jessup RL, Osborne RH, Buchbinder R, et al. Using co-design to develop interventions to address health literacy needs in a hospitalised population. BMC Health Serv Res 2018;18:989.

3 Banbury A, Nancarrow S, Dart J, et al. Adding value to remote monitoring: Co-design of a health literacy intervention for older people with chronic disease delivered by telehealth - The telehealth literacy project. Patient Educ Couns 2020;103:597-606.

4 New South Wales Government. A guide to build Co-design capability Chatswood NSW 2067: agency for clinical innovation. New South Wales Government, 2019.

5 Slattery P, Saeri AK, Bragge P. Research co-design in health: a rapid overview of reviews. Health Res Policy Syst 2020;18:17.

6 Chalmers I, Glasziou P. Avoidable waste in the production and reporting of research evidence. Lancet 2009;374:86-9.

7 loannidis JPA. Why most clinical research is not useful. PLoS Med 2016;13:e1002049.

8 Anderson GF. Missing in action: international aid agencies in poor countries to fight chronic disease. Health Aff 2009;28:202-5.

9 Yadav UN, Lloyd J, Hosseinzadeh H, et al. Facilitators and barriers to the self-management of COPD: a qualitative study from rural Nepal. BMJ Open 2020;10:e035700.

10 Dhimal M, Karki KB, Sharma SK, et al. Prevalence of selected chronic non-communicable diseases in Nepal. J Nepal Health Res Counc 2019;17:394-401.

11 Yadav UN, Lloyd J, Hosseinzadeh H, et al. Self-Management practice, associated factors and its relationship with health literacy and patient activation among multi-morbid COPD patients from rural Nepal. BMC Public Health 2020;20:300.

12 Yadav UN, Lloyd J, Hosseinzadeh $\mathrm{H}$, et al. Levels and determinants of health literacy and patient activation among multi-morbid COPD people in rural Nepal: findings from a cross-sectional study. PLoS One 2020;15:e0233488.

13 Boaz A, Hanney S, Borst R, et al. How to engage stakeholders in research: design principles to support improvement. Health Res Policy Syst 2018;16:60.

14 Fucile B, Bridge E, Duliban C, et al. Experience-Based co-design: a method for patient and family engagement in system-level quality improvement. Patient Exp J 2017;4:53-60.

15 Dimopoulos-Bick T, Dawda P, Maher L, et al. Experience-Based CoDesign: tackling common challenges. The Journal of Health Design 2018;3:86-93. 
16 Plattner H. An introduction to design thinking process guide. USA: Hasso Platter Institute of Design at Stanford.

17 Plattner H, Meinel C, Leifer L. Design Thinking: Understand - Improve - Apply. Springer Publishing Company, Incorporated, 2010.

18 Knoblauch H. Focused ethnography. forum. Qualitative Social Research 2005;6:44

19 Gruman J, Rovner MH, French ME, et al. From patient education to patient engagement: implications for the field of patient education. Patient Educ Couns 2010;78:350-6.

20 Baumbusch J, Wu S, Lauck SB, et al. Exploring the synergies between focused ethnography and integrated knowledge translation. Health Res Policy Syst 2018;16:103.

21 Stevahn L, King JA. Facilitating interactive evaluation practice: engaging stakeholders Constructively. New Dir Eval 2016;2016:67-80.

22 PJJ HGM, Boadu NY. Guidance on performing focused Ethnographies with an emphasis on healthcare research. The qualitative report, 2013.

$23 \mathrm{~K} \mathrm{H}$. Focused ethnography. forum qualitative Sozialforschung / forum. Qualitative Social Research 2005;6.

24 Maxwell J. Qualitative Research Design : An Interactive Approach. J. A. Maxwell, 2012.

25 Braun V, Clarke V. Using thematic analysis in psychology. Qual Res Psychol 2006;3:77-101.

26 Bate P, Robert G. Experience-Based design: from redesigning the system around the patient to co-designing services with the patient. Qual Saf Health Care 2006;15:307-10.

27 Maher LM, Hayward B, Hayward P, et al. Increasing patient engagement in healthcare service design: a qualitative evaluation of a co-design programme in New Zealand. Patient Exp $J$ 2017;4:23-32.

28 Bowen S, Sustar H, Wolstenholme D, et al. Engaging teenagers productively in service design. Int $J$ Child Comput Interact 2013;1:71-81.

29 Nakarada-Kordic I, Hayes N, Reay SD, et al. Co-designing for mental health: creative methods to engage young people experiencing psychosis. Design for Health 2017;1:229-44.

30 Forsythe LP, Ellis LE, Edmundson L, et al. Patient and Stakeholder engagement in the PCORI pilot projects: description and lessons learned. J Gen Intern Med 2016;31:13-21.

31 Camden C, Shikako-Thomas K, Nguyen T, et al. Engaging stakeholders in rehabilitation research: a scoping review of strategies used in partnerships and evaluation of impacts. Disabil Rehabil 2015;37:1390-400.

32 Bailey S, Boddy K, Briscoe S, et al. Involving disabled children and young people as partners in research: a systematic review. Child Care Health Dev 2015;41:505-14.

33 Puts MTE, Hardt J, Monette J, et al. Use of geriatric assessment for older adults in the oncology setting: a systematic review. J Nat Cancer Inst 2012;104:1134-64.

34 Yamey G. What are the barriers to scaling up health interventions in low and middle income countries? A qualitative study of academic leaders in implementation science. Global Health 2012;8:11.

35 Goodyear-Smith F, Jackson C, Greenhalgh T. Co-design and implementation research: challenges and solutions for ethics committees. BMC Med Ethics 2015;16:78.
36 Janamian T, Jackson CL, Dunbar JA. Co-creating value in research: stakeholders' perspectives. Med J Aust 2014;201:S44-6.

37 Aarons GA, Hurlburt M, Horwitz SM. Advancing a conceptual model of evidence-based practice implementation in public service sectors. Adm Policy Ment Health 2011;38:4-23.

38 Piña IL, Cohen PD, Larson DB, et al. A framework for describing health care delivery organizations and systems. Am J Public Health 2015;105:670-9.

39 Esmail L, Moore E, Rein A. Evaluating patient and stakeholder engagement in research: moving from theory to practice. J Comp Eff Res 2015;4:133-45.

40 Oliver SR, Rees RW, Clarke-Jones L, et al. A multidimensional conceptual framework for analysing public involvement in health services research. Health Expect 2008;11:72-84.

41 O'Brien J, Fossey E, Palmer VJ. A scoping review of the use of codesign methods with culturally and linguistically diverse communities to improve or adapt mental health services.

42 Peters DH, Bhuiya A, Ghaffar A. Engaging stakeholders in implementation research: lessons from the future health systems research programme experience. Health Res Policy Syst 2017;15:104.

43 Gustavsson SMK, Andersson T. Patient involvement 2.0: experiencebased co-design supported by action research. Action Res 2019;17:469-91.

44 Stacciarini J-MR, Shattell MM, Coady M, et al. Review: communitybased participatory research approach to address mental health in minority populations. Community Ment Health J 2011;47:489-97.

45 Domecq JP, Prutsky G, Elraiyah T, et al. Patient engagement in research: a systematic review. BMC Health Serv Res 2014;14:89.

46 Guise J-M, O'Haire C, McPheeters M, et al. A practice-based tool for engaging stakeholders in future research: a synthesis of current practices. J Clin Epidemiol 2013;66:666-74.

47 Haijes HA, van Thiel GJMW. Participatory methods in pediatric participatory research: a systematic review. Pediatr Res 2016;79:676-83.

48 Ward ME, De Brún A, Beirne D, et al. Using Co-Design to develop a collective leadership intervention for healthcare teams to improve safety culture. Int J Environ Res Public Health 2018;15:118.

49 Hjelmfors L, Strömberg A, Friedrichsen M, et al. Using co-design to develop an intervention to improve communication about the heart failure trajectory and end-of-life care. BMC Palliat Care 2018;17:85.

50 Service NH. Improvement Leaders' Guide: Sustainability and its Relationship with Spread and Adoption. Institute for Innovation and Improvement: NHS, 2010.

51 Damschroder LJ, Aron DC, Keith RE, et al. Fostering implementation of health services research findings into practice: a consolidated framework for advancing implementation science. Implement Sci 2009;4:50.

52 May CR, Johnson M, Finch T, Implementation FT. Implementation, context and complexity. Implement Sci 2016;11:141.

53 Earle L, Earle T. Social context theory: a systems model of social change based on social needs and social issues. South Pacific Journal of Psychology 1999;11:1-12. 